\title{
The Effect of the Stiffness Gradient on the Just Noticeable Difference Between Surface Regions
}

\author{
Umut Koçak, Karljohan Lundin Palmerius, Camilla Forsell, and \\ Matthew Cooper \\ C-Research, Linköping University, SWEDEN \\ \{umut.kocak, karljohan.lundin.palmerius, camilla.forsell, matthew. cooper\}@ \\ liu.se
}

\begin{abstract}
Numerous studies have considered the ability of humans to perceive differences in forces and how this affects our ability to interpret the properties of materials. Previous research has not considered the effect of the rate of change of the material stiffness in our ability to perceive differences, however, an important factor in exploration processes such as a doctor's palpation of the skin to examine tissues beneath. These effects are the topic of this research which attempts to quantify the effects of stiffness gradient magnitude and form on the discernment of changes in stiffness.
\end{abstract}

Keywords: Perception, stiffness, gradient, exploratory procedures, JND

\section{Introduction}

Many disciplines have contributed to research in haptics in the last decade. One of them is psychophysics which has focussed on understanding our perception mechanism in order to facilitate the design of more effective haptics hardware and software solutions in a wide range of application areas. An ever increasingly important application for haptic technologies is surgery simulation where the feedback increases the chance of transferring knowledge and skills between the simulated and real environment.

One of the major aspects of exploring tissues and understanding the properties of the material at hand is its hardness/softness. Yet surgery simulation is but one example of haptic interaction with compliant surfaces, so it is to be expected that stiffness/compliance will be one of the most frequently surveyed material properties with several studies (for example [12]) being conducted on just noticeable difference (JND) within different scenarios and ranges. In our opinion, however, the multifaceted nature of stiffness exploration has only been explored for one type of interaction. The continuity of the contact and stiffness changes during continuous contact have not been surveyed comprehensively, despite their frequent importance in real life scenarios. During tissue palpation, for example searching for malign growth beneath the skin, the perception of stiffness change during contact is crucial since the changes reflect the material properties of the different tissues present. Several studies in the medical field [4, 
$15,16]$ have been conducted showing the direct relationship between tissue characteristics and the stiffness gradient, yet its implications for human perception of stiffness are largely untested.

In this paper we present a study of the effect of stiffness gradient on the perception of stiffness in materials. Such a study can capture the effects of the rate of change of the stiffness during continuous contact as experienced in such exploratory procedures as drilling, cutting or palpation. Previous work [5] has demonstrated the effects of continuous contact, and axis of contact, on the JND in the stiffness but the effects of the rate of change have not been previously considered in perception studies, to the authors' knowledge. Our experiments, carried out within a virtual environment, have been conducted in order to demonstrate and measure the effect of stiffness gradient on discrimination. The results show not only an effect upon the observable difference between stiffness levels but also that the stiffness gradient affects the discrimination during continuous contact.

\section{Related Work}

Stiffness (or compliance) is one of the most studied properties, representing the hardness or softness of an object. The most common means to explore stiffness perception is to present a measure, showing how well humans can perceive the varying levels of hardness or softness, in the form of a JND. The results found vary depending on the differences between the methods employed. Effects of other factors such as multi-modality, and cutaneous and kinaesthetic cues are also being surveyed.

There are a number of studies (for example [12]) which have examined the JND in the stiffness. The effects of force and work cues on compliance discrimination were surveyed in [13] and the significant effect of force cues on discrimination was evident. In [14], the effect of surface deformation cues was examined and it was shown that the subjects' ability to discriminate the difference in stiffness was reduced by a factor of more than three without deformation cues.

The effect of visual information on stiffness perception was explored in the studies $[10,17]$. The dominance of visual feedback over kinaesthetic sense of hand position was demonstrated in [10]. Compliant objects that are further away were perceived to be softer in the case of haptic feedback alone [17], while the addition of the visual information reduced this bias.

Further studies $[3,6,11]$ have examined the effects of some of the exploratory procedures [7] on stiffness perception. These exploratory procedures directly affect which properties of the object can be observed and how we perceive them. In [11], the contribution of tactile and kinaesthetic cues were explored for deformable and non-deformable objects. It was shown that the tactile information alone is sufficient for discrimination capacity of deformable objects while additional kinaesthetic feedback is necessary for non-deformable compliant objects. When a tool was used for exploration, additional kinaesthetic cues were found to be necessary for all types of objects [6]. Squeezing a deformable object between

CSpringer-Verlag 2012. This is the autRors version of the work. It is posted here by permission of Springer for your personal use. Not for redistribution. The original publication is available at www.springerlink.com 

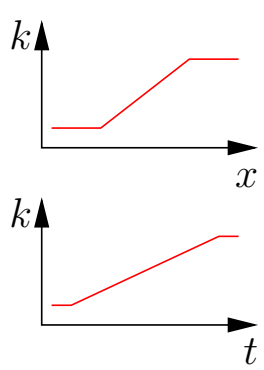

(a)
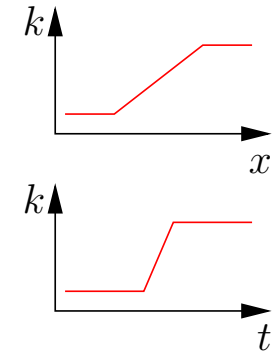

(b)
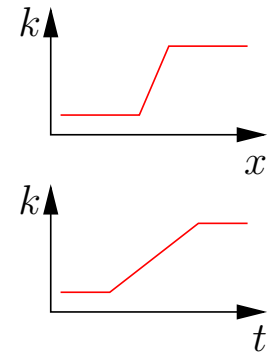

(c)
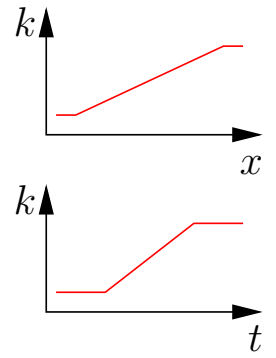

(d)

Fig. 1. The stiffness gradient over space (top) and over time (bottom). In subfigures (a) and (b) the position dependent stiffness over the transition region is the same, with low hand velocity (a) and high hand velocity (b) producing different perceived temporal changes in stiffness. In subfigures (c) and (d), the time dependent stiffness over the transition region is the same, with low hand velocity (c) and high hand velocity (d) producing different perceived spatial changes.

thumb and index finger was explored in $[3,9]$ and tactile information was found to be negligible for this scenario [9].

The studies described above surveyed the perception of stiffness and some affecting factors such as the visual information or exploratory procedure. The discrimination during continuous contact has been surveyed in [5] but, to the knowledge of the authors, the stiffness gradient has never been considered in discrimination studies.

\section{Stiffness Change During Contact}

The exploration of stiffness change during retained contact considered in this paper requires at least a $\mathrm{C}^{0}$ continuous stiffness function. There are, however, two ways to describe the stiffness change during contact: stiffness as a function of position and stiffness as a function of time. The former is naturally what a real interaction would express. Nevertheless, for the proper understanding of the actual effect time may have a significant impact, as is discussed below.

\subsection{Time-Dependent and Position-Dependent Change}

The velocity of the hand during contact is one of the factors affecting the stiffness characteristics such that different hand velocities result in different stiffness-time or stiffness-position functions, as illustrated in figure 1. One can think of the scenario in figures $1(\mathrm{a})$ and $1(\mathrm{~b})$ as stiffness perceived during a lateral movement across a flat surface composed of two regions exhibiting different stiffnesses with a transition region between them. The graphs at the top refer to the stiffness change across the flat surface. If one makes a lateral movement on the surface from one side to the other, lower hand velocity would create an apparently

(C) Springer-Verlag 2012. This is the autBors version of the work. It is posted here by permission of Springer for your personal use. Not for redistribution. The original publication is available at www.springerlink.com 
smoother stiffness change with time, as shown at the bottom graph in figure 1(a), while a higher hand velocity would correspond with the bottom graph in figure 1 (b) which shows a sharper stiffness change in time. The position-stiffness characteristics are not affected by the hand velocity in this case.

Alternatively, if the stiffness is controlled by time, and independent of position, another scenario arises (see figures $1(\mathrm{c})$ and $1(\mathrm{~d})$ ). For a continuous lateral motion across the flat surface with a reference hand velocity, the stiffness-time and stiffness-position graphs would be the same. A lower velocity would create the stiffness-position graph at the top in figure 1(c) with an apparently sharper stiffness change over space while a higher velocity would create the stiffnessposition graph at the top in figure $1(\mathrm{~d})$ with an apparently more gradual stiffness change over space.

The effect of these parameters need to be understood for the design of the full study presented in this paper so a pilot study was conducted, exploring the effects of the two scenarios in figure 1 with respect to the JND. During the pilot studies the subject was asked to make a lateral movement across a virtual surface while matching the speed indicated by an animation of a set of balls moving between sides with a constant velocity, figure $3(\mathrm{~b})$. The subject was not expected to exactly match the velocity of the moving balls, the aim was instead to provide an approximate guidance of the movement of the subject's hand. A JND of stiffness was found for the position-dependent and time-dependent stiffness conditions for two different hand velocities, $2 \mathrm{~cm} / \mathrm{sec}$ and $8 \mathrm{~cm} / \mathrm{sec}$, by one subject and with 3 repetitions. In the case of the position-dependent stiffness the JND was smaller for higher hand velocity cases, creating a sharper stiffness change in the time domain, in 2 out of 3 repetitions. For the time dependent stiffness the JND was smaller in lower velocity cases, creating a sharper stiffness change in the position domain, in all repetitions. As a result, the pilot studies hinted at possibly better discrimination performance with a higher stiffness gradient and showed that we need to consider the hand velocity during contact since both the stiffness-time and stiffness-position functions have the potential to affect the perception.

\subsection{Transition Region}

To examine the effect of the stiffness gradient on stiffness discrimination, three conditions with different position dependent stiffness functions were considered. All of the stiffness functions included two regions exhibiting different, constant stiffness values with a transition region between them. The two stiffness values and the width of the transition region were chosen to be the same for all three conditions. The difference between the stiffness levels, $\Delta_{\mathrm{k}}$, the transition width, $\Delta_{\mathrm{x}}$ and the stiffness gradients are illustrated in figure 2 . The three conditions differ in the interpolation function used in the transition region. The three interpolation functions for the conditions are linear, cosine, and tanh each of which have different derivatives. This results in a different maximum magnitude of stiffness gradient with transition region size and stiffness difference kept the same.

(C)Springer-Verlag 2012. This is the authors version of the work. It is posted here by permission of Springer for your personal use. Not for redistribution. The original publication is available at www.springerlink.com 


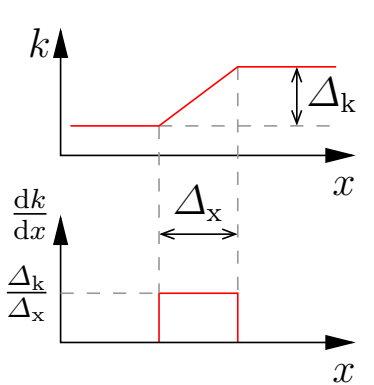

(a) Linear interpolation.

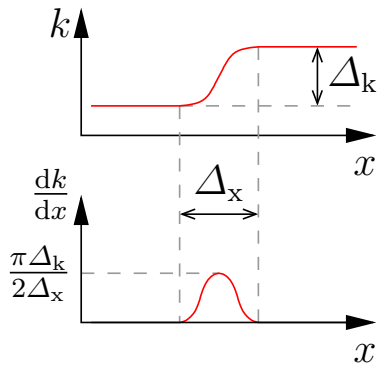

(b) Cosine interpolation.

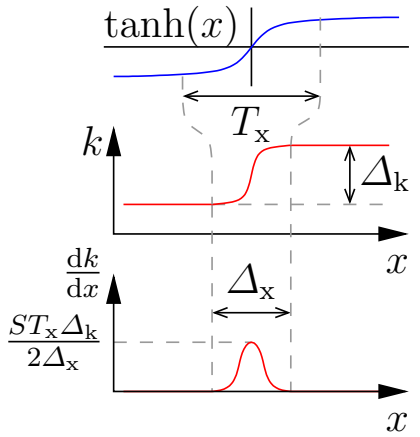

(c) Tanh interpolation.

Fig. 2. The interpolation functions used in the study, with their respective spatial derivatives.

In the case of linear interpolation the gradient is a constant function with a value of $\Delta_{\mathrm{k}} / \Delta_{\mathrm{x}}$ in the transition region. In the second condition a cosine function is mapped by

$$
k(x)=\frac{\Delta_{\mathrm{k}}}{2} \cos \left(\pi\left(\frac{x-x_{0}}{\Delta_{\mathrm{x}}}\right)-\pi\right)
$$

with a derivative

$$
\frac{\mathrm{d} k}{\mathrm{~d} x}(x)=\frac{-\Delta_{\mathrm{k}}}{\Delta_{\mathrm{x}}} \frac{\pi}{2} \sin \left(\pi\left(\frac{x-x_{0}}{\Delta_{\mathrm{x}}}\right)-\pi\right)
$$

where $x_{0}$ is the beginning point of the transition region. It can be seen from equation 2 that the maximum stiffness gradient in the transition region is $\pi / 2$ times the linear case. The tanh function, which converges to 1 and -1 at infinity and negative infinity respectively, was chosen as a third condition. By mapping a limited range of the tanh function around the origin to the transition region one can adjust the maximum stiffness gradient in the transition region. The function must be stretched by a factor, $\mathrm{S}$, to make it $\mathrm{C}^{0}$ continuous in the edge between the transition region and the respective adjacent constant regions. The tanh function is thus mapped to the transition region by

$$
k(x)=\frac{S \Delta_{\mathrm{k}}}{2} \tanh \left(T_{\mathrm{x}}\left(\frac{x-x_{0}}{\Delta_{\mathrm{x}}}\right)-\frac{T_{\mathrm{x}}}{2}\right)
$$

where

$$
S=\frac{1}{\tanh \left(\frac{T_{x}}{2}\right)}
$$

with a derivative

$$
\frac{\mathrm{d} k}{\mathrm{~d} x}(x)=\frac{S \Delta_{\mathrm{k}}}{\Delta_{\mathrm{x}}} \frac{T_{\mathrm{x}}}{2} \frac{1}{\cos ^{2}\left(T_{\mathrm{x}}\left(\frac{x-x_{0}}{\Delta_{\mathrm{x}}}\right)-\frac{T_{\mathrm{x}}}{2}\right)}
$$

(C)Springer-Verlag 2012. This is the authors version of the work. It is posted here by permission of Springer for your personal use. Not for redistribution. The original publication is available at www.springerlink.com 


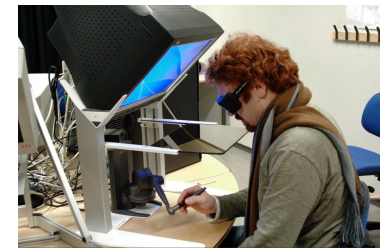

(a)

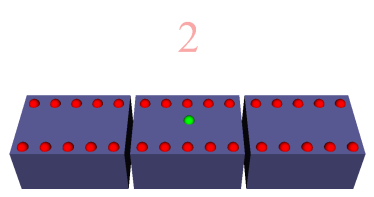

(b)

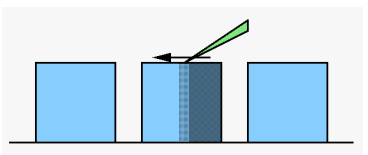

(c)

Fig. 3. (a) A $3 \mathrm{D}$ virtual image registered with the real hand position is obtained with the help of a semi-transparent mirror and stereo glasses. (b) An animation of a series of red balls moving to the left and to the right were rendered at the front and back of the boxes, respectively. (c) Trials included three boxes, only one of which included a stiffness change along lateral motion across the surface.

Deploying three different interpolation functions allows comparison of three different stiffness gradient magnitudes while keeping the transition width and the stiffness difference identical for all three conditions. The maximum stiffness gradient is $\pi / 2$ times and $S T_{\mathrm{x}} / 2$ times the gradient of the linear condition in the cosine and tanh conditions, respectively.

\section{Evaluation}

To explore the effect of stiffness gradient we performed a user study in two stages: gradient magnitude and transition width. In the gradient magnitude case, we aimed to test the effect of gradient magnitude by comparing the three different interpolation functions in the transition region. In the second stage, different transition widths were presented and the psychometric function of the gradient was measured from the subjects' responses.

\subsection{Method}

The experiments were performed in a virtual environment and a Desktop Phantom and a semi-transparent framework was used as the equipment, as illustrated in figure 3(a). Each stimulus was composed of three virtual boxes that were visually rendered as in figure 3(b). The orientation of the boxes was adjusted such that the palpation occurs on the axis perpendicular to the desk. To prevent visual cues relating to the strain applied, the appearance of the boxes did not change in response to the compression and the haptic probe was rendered as a sphere which remained on the surface of the boxes during contact in all situations. The subject was also prevented from seeing the real hand position under the semi-transparent mirror by installing a sheet of white paper under the mirror and setting the background colour to bright white. In order to have the subjects adopt approximately the same hand velocity during lateral movement, an animation of a set of red balls moving to right and left were rendered at the front and back of the boxes, respectively. Two of the boxes were identical while

(C)Springer-Verlag 2012. This is the aut6iors version of the work. It is posted here by permission of Springer for your personal use. Not for redistribution. The original publication is available at www.springerlink.com 
one of them, selected at random, had a non-uniform stiffness as described below. The force feedback from the boxes was evaluated by multiplying the stiffness and the depth of probe from the surface of the box, based on Hooke's Law. The subjects were told to make 'sweeping' movements sideways (left-to-right-to-left) across the surface, following the speed of the ball animation, and select the box which exhibited non-uniform stiffness in each trial.

In all trials in both experiments, the subjects were presented with three different boxes two of which presented only the reference stiffness while the third, selected at random, presented the reference stiffness on the left half side and a harder stiffness on the right with a transition region in between them, see figure $3(\mathrm{c})$. The height of the boxes was set to $3 \mathrm{~cm}$ throughout the experiments. In the literature a wide range of reference stiffnesses, varying from $100 \mathrm{~N} / \mathrm{m}$ to 16900 N/m [3], have been surveyed. During several pilot studies various stiffness values had been tested. It was observed that continuous use of the haptic device with higher stiffness values can result in overheating of the motors, requiring a break for the system to cool down. Finally, $100 \mathrm{~N} / \mathrm{m}$ was determined as a suitable reference stiffness.

Twelve subjects took part in the experiments, 9 male and 3 female. They were all undergraduate or graduate students aged between 23 and 40 years (mean age was 28). 10 of the subjects had tried a haptic device on a few occasions previous to the experiments and 2 had used them quite often. All subjects had normal or corrected to normal vision. They received no compensation for taking part in the experiments.

Before the experiments began background information was obtained from each subject. They then reviewed written instruction material and were instructed about the equipment and the tasks to be performed. Before the real experiments they also completed a set of practice trials. For each individual trial the task was to identify which box, out of the three, exhibited the different stiffness levels and give a response by pressing a button placed on the haptic device while pointing to that box. Total participation time lasted 1 hour, on average, including the introductory part.

\subsection{Gradient Magnitude Evaluation}

The JNDs of three conditions with different interpolation functions in the transition region of the non-uniform box, figure 3(c), were compared. Having a better discrimination performance in one of these conditions, being able to detect smaller $\Delta_{\mathrm{k}}$, would indicate the contribution from another component. We suspected that the presence of a higher stiffness gradient would contribute to the sense of discrimination and so result in a smaller JND. Therefore we compared the JNDs of the three conditions depending on the interpolation function used in the transition region: Linear, cosine and tanh functions (as described in section 3.2).

For all the three conditions, the magnitude of the harder stiffness in the nonuniform box was changed depending on the subjects' previous responses, while the reference stiffness was kept constant. A one-up two-down adaptive staircase

(C)Springer-Verlag 2012. This is the authors version of the work. It is posted here by permission of Springer for your personal use. Not for redistribution. The original publication is available at www.springerlink.com 
procedure was used [8] while changing the harder stiffness. An adaptive staircase starts with an initial difference magnitude and, depending on an individual subject's responses, changes the magnitude of the difference such that it converges to the perception limit of discrimination for that subject. In the case of a one-up two-down staircase, the magnitude is decreased following two consecutive correct responses and increased after each single incorrect response. This procedure converges to a stimulus level at which subjects can make accurate responses with a certainty of $70.7 \%$. In our case each session started with a stiffness difference of $66.7 \mathrm{~N} / \mathrm{m}(2 / 3$ of the reference stiffness value). Initially, the stiffness difference was changed by $9 \mathrm{~N} / \mathrm{m}$ per response and then by $4.5 \mathrm{~N} / \mathrm{m}$ after the third reversal and by $2.25 \mathrm{~N} / \mathrm{m}$ after the sixth reversal. The session was terminated after nine reversals and the average of the peaks and valleys of the last six reversals were calculated to be the JND. The width of the transition region was set to $10 \mathrm{~mm}$ for all conditions. For the tanh condition, the mapped range of the tanh function, $T_{\mathrm{x}}$, was set to 6 .

The evaluation was performed as a within-subjects design with one independent variable (stiffness) having three interpolation functions (Linear vs. Cosine vs. Tanh) or conditions. The experiment was performed over three separate sessions where each condition was carried out once. The presentation order of the conditions for each subject was balanced by using a Latin-square procedure. The placement of the box with variable stiffness was randomized for each trial.

\subsection{Transition Width Evaluation}

In the second stage, an experiment was performed by each subject in order to calculate the probabilities of the correct guesses for different transition region widths. In this stage, the stiffness difference was kept constant at the JND value for each subject, as found using the linear condition in the first stage at a transition width of $10 \mathrm{~mm}$. The width of the transition region was set to four different values: $1.25,5,20$ and $80 \mathrm{~mm}$. Each transition width was repeated 10 times in a random order, with the linear transition function used throughout. These repetitions were used to calculate the probability of a correct response for each width.

\section{Results}

The values for each of the 12 subjects for all three conditions in the first stage were analyzed. According to the Kolmogorov-Smirnov and Shapiro-Wilks tests, the data did not fit a normal curve therefore we employed a logarithmic transformation which corrected the fit. Hence we used a parametric test for further analysis. A repeated measures ANOVA with a decision criterion of 0.05 showed that there was a significant difference between the three conditions, $\mathrm{F}(2,22)=$ 9.059, $\mathrm{p}=0.001$.

To determine which conditions significantly differed from each other, Bonferroni corrected pairwise comparisons were performed at a $0.0167(0.05 / 3)$ level

(C)Springer-Verlag 2012. This is the aut\$ors version of the work. It is posted here by permission of Springer for your personal use. Not for redistribution. The original publication is available at www.springerlink.com 


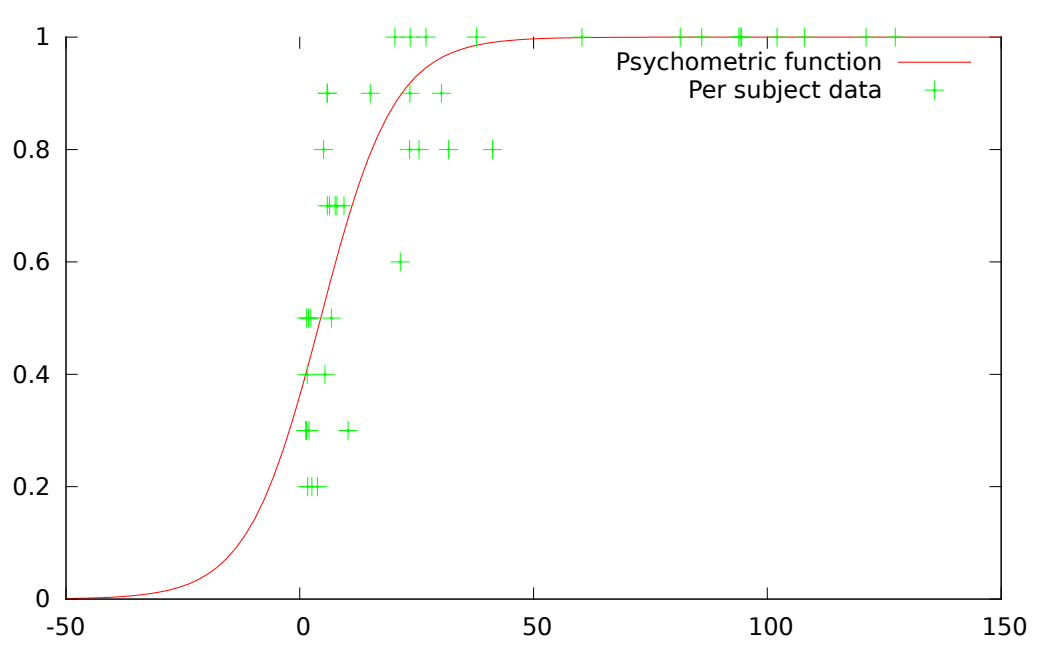

Fig. 4. The probability of a correct response as a function of the stiffness gradient $(\mathrm{N} / \mathrm{m}$ per $\mathrm{cm}$ ).

of significance as a post-hoc test. A significant difference was observed between condition tanh and the other two conditions, $\mathrm{p}<0.05$, while no significant difference was observed between the conditions cosine and linear, $\mathrm{p}>0.05$. The significant difference for the tanh condition was more likely since the maximum stiffness gradient is $S T_{\mathrm{x}} / 2$, approximately three times the gradient in the linear condition, where $\mathrm{S}$ is close to 1 and $T_{\mathrm{x}}$ was chosen to be 6 . Not having a significant difference between the cosine and linear conditions might be explained by the closer maximum stiffness gradient values. Maximum gradient of the cosine condition was $\pi / 2$ (approximately 1.6) times that of the linear condition.

The analysis of the results shows that the discrimination performance for the condition with the highest stiffness gradient magnitude in the transition region, tanh, is significantly better than the other two conditions. The mean value of the JND for the cosine condition is $14.7 \pm 8.9 \%$, while the linear and tanh conditions have a mean JND of $17.95 \pm 9.62 \%$ and $10.86 \pm 6.24 \%$ respectively.

The probabilities of correct responses found in the second stage experiment are illustrated in figure 4 . One can observe the characteristic psychometric function [8] for a range of the stiffness gradient showing strong correlation between gradient and discrimination. The graph extends into negative (imaginary) widths because of the risk of missing the small stiffness difference even at a small transition width. A least-squares fitted sigmoid curve shows that the average $50 \%$ probability lies at approximately $4,5 \mathrm{~N} / \mathrm{m}$ per $\mathrm{cm}$, but also that the individual data differs substantially compared to this average.

(C) Springer-Verlag 2012. This is the authors version of the work. It is posted here by permission of Springer for your personal use. Not for redistribution. The original publication is available at www.springerlink.com 


\section{Discussion}

We performed experiments in order to observe the effect of stiffness gradient on discrimination of the stiffness change during continuous contact. We compared the JNDs of three conditions with different interpolation functions deployed in the transition between two stiffness levels. The interpolation functions differed in their stiffness gradient magnitude and a significantly better discrimination was obtained for the one with highest gradient magnitude. Among the other two conditions the discrimination appeared to be better for the higher gradient magnitude condition but the results were not statistically significant.

The results being consistent with the relation that the higher the stiffness gradient the better the discrimination, support the idea that it is not only stiffness difference but also the gradient affecting the perception. This was also supported by the second stage of the experiment, performed to achieve the psychometric function showing the probability of correct responses in the discrimination task as a function of stiffness gradient.

In [2], it was discussed that observing a Weber fraction for a continuous signal, the percentage change in the signal that can be barely noticed, depends on the type of the haptic signal. Stiffness was among the signals for which the Weber fraction has often been observed, making the stiffness perception context-dependent. This context-dependency is also well-known for the perception of colour [1], due to perceiving the same colour differently depending on the surrounding colours within which it is presented. Our results, by showing the relationship between the stiffness gradient and the discrimination, also indicate the importance of the context - the type of the change in addition to the magnitude of the change.

Discrimination of stiffness change might be crucial in real life scenarios such as exploring tissues for malign growth, needle insertion, and tissue cutting and bone drilling in surgeries. Understanding our perception mechanism and its limits has the potential of improving the procedures in real life, in addition to improving virtual medical applications such as surgery simulators. The knowledge of the perception of continuous stiffness change can also be useful for piecewise linear modelling of nonlinear stiffness for providing smoother representations of the nonlinearity.

We think of this result as a starting point for further examination of stiffness perception for scenarios closer to real life situations. Studies [3, 6, 11] of the effect of exploratory procedures on perception have shown the need to design more realistic scenarios in perception studies to achieve useful results. In addition to surveying different aspects of perception during continuous touch, we intend to explore ways to combine these results in our haptic software solutions as well.

\section{Acknowledgments}

This work has been funded by the Swedish Science Council through grant number 621-2005-3609, the Foundation for Strategic Research (SSF) under the Strategic

(C)Springer-Verlag 2012. This is the autloors version of the work. It is posted here by permission of Springer for your personal use. Not for redistribution. The original publication is available at www.springerlink.com 
Research Center MOVIII, and the Swedish Research Council Linnaeus Center CADICS.

\section{References}

1. Adelson, E.H.: Lightness Perception and Lightness Illusions. In: The New Cognitive Neurosciences, 2nd ed., pp. 339-351. Cambridge, MA:MIT Press, (2000)

2. Cholewiak, S.A., Tan, H.Z., Ebert, D.S.: Haptic Identification of Stiffness and Force Magnitude. In: Haptic Interfaces for Virtual Environment and Teleoperator systems, pp. 87-91. IEEE Press, (2008)

3. Freyberger, F.K., Frber, B.: Compliance discrimination of deformable objects by squeezing with one and two fingers. In: Eurohaptics, pp. 271-276. (2006)

4. Isenberg, B.C., Dimilla, P.A., Walker, M., Kim, S., Wong, J.Y.: Vascular Smooth Muscle Cell Durotaxis Depends on Substrate Stiffness Gradient Strength. Biophysical Journal. 97, 1313-1322 (2009)

5. Kocak, U., Palmerius, K.L., Forsell, C., Ynnerman, A., Cooper, M.: Analysis of the JND of Stiffness in Three Modes of Comparison. In: Haptic and Audio Interaction Design, pp. 22-31. (2011)

6. Lamotte, R.H.: Softness discrimination with a tool. J. Neurophysiology. 83(4), 1777$1786(2000)$

7. Lederman, S., Klatzky, R.: Haptic perception: A tutorial. Attention, Perception, and Psychophysics 71(7), 1439-1459 (2009)

8. Levitt, H.: Transformed up-down methods in psychoacoustics. J. Acoustical Society of America. 49, 467-477 (1971)

9. Roland, P., Ladegaard-Pedersen, H.: A quantitative analysis of sensations of tension and of kinasthesia in man: Evidence for a peripherally originating muscular sense and for a sense of effort. Brain. 100(4), 671-692 (1977)

10. Srinivasan, M.A., Beauregard, G., Brock, D.: The impact of visual information on the haptic perception of stiffness in virtual environments. In: ASME Dynamic Systems and Control Division, pp. 555-559. (1996)

11. Srinivasan, M.A., Lamotte, R.H.: Tactual discrimination of softness. J. Neurophysiology. 73(1), 88-101 (1995)

12. Tan, H.Z., Pang, X.D., Durlach, N.I.: Manual resolution of length, force and compliance. In: ASME Dynamic Systems and Control Division, pp. 13-18. (1992)

13. Tan, H.Z., Durlach, N.I., Beauregard, G., Srinivasan, M.A. : Manual discrimination of compliance using active pinch grasp: The roles of force and work cues. Perception and Psychophysics. 57(4), 495-510 (1995)

14. Tiest, W.M.B., Kappers, A.M.: Cues for haptic perception of compliance. IEEE Transactions on Haptics. 2(4), 189-199 (2009)

15. Tse, J.R., Engler, A.J.: Stiffness Gradients Mimicking In Vivo Tissue Variation Regulate Mesenchymal Stem Cell Fate. PLoS ONE. 6(1), e15978 (2011)

16. Wong, J.Y., Velasco, A., Rajagopalan, P., Pham, Q.: Directed Movement of Vascular Smooth Muscle Cells on Gradient-Compliant Hydrogels. Langmuir. 19(5), 19081913 (2003)

17. Wu, W.C., Basdogan, C., Srinivasan, M.A.: Visual, haptic, and biomodal perception of size and stiffness in virtual environments. In: ASME Dynamic Systems and Control Division, pp. 19-26. (1999)

(C)Springer-Verlag 2012. This is the authlors version of the work. It is posted here by permission of Springer for your personal use. Not for redistribution. The original publication is available at www.springerlink.com 